\title{
DEPRESIÓN Y AUTOESQUEMAS DEPRESIVOS EN PACIENTES DEPRIMIDOS Y ANSIOSOS
}

\author{
$M^{2}$ Ángeles Ruipérez' y Amparo Belloch ${ }^{2}$ \\ ${ }^{1}$ Universitat Jaume I. (Castellón) \\ 2 Universitat de Valencia
}

\begin{abstract}
RESUMEN
Uno de los modelos psicológicos más influyentes para el estudio de los trastornos emocionales es el propuesto por Beck, quien propone que los autoesquemas de las personas deprimidas contienen generalizaciones de caracteristicas predominantemente negativas sobre el si-mismo. La existencia de autoesquemas negativos en la depresión se ha estudiado mediante procedimientos como la "Tarea de codificación autorreferente" (TCA) utilizando casi siempre poblaciones subclínicas y/o muestras control de personas normales. Este trabajo tiene por objeto estudiar la existencia de autoesquemas negativos en pacientes clínicamente deprimidos. La muestra de estudio estuvo formada por 22 pacientes con diagnóstico de Depresión Mayor, 17 Distímicos y 39 de Ansiedad Generalizada. Los resultados apoyan solo parcialmente la hipótesis acerca de un autoesquema predominantemente negativo en la depresión. Los pacientes con diagnóstico de depresión mayor presentaron en efecto un esquema cuyo contenido era básicamente negativo, pero no hubo diferencias notables entre distímicos y ansiosos. Estos resultados ponen en duda la generalización de los datos obtenidos en poblaciones no clínicas a contexos clínicos reales, al menos por lo que se refiere a los trastornos emocionales. Además, la consideración de los trastomos del estado de ánimo como una entidad unitaria y diferente de los trastomos de ansiedad, queda en entredicho cuando se utilizan estrategias experimentales como la TCA.
\end{abstract}

Palabras clave: Depresión, Ansiedad, procesos cognitivos, trastornos emocionales, autoesquema, tarea de codificación autorreferente.

\begin{abstract}
The Beck model is one of the most currently influential psychological frameworks to the study of the emotional disorders. From this perspective the self-schemas of depressed persons are characterised by a predominantly negative content. In this paper we have studied the existence, content and specificity of such negative self-schemas in depression using the self-referent encoding task (SRET). Twenty-two major depressed, 17 dysthymic, and 39 generalized anxiety patients performed the SRET. The results partially support the existence of a generalised negative self-schema in depressed patients. Major depressed patients showed a more negative self-schema than the other two groups of patients. However, dysthymic and anxious patients do not exhibited a consistently different performance pattern. These findings are discussed in relation to the lack of generalizability to the clinical population of the results obtained when normal and/or subclinical samples are used to test the cognitive models of the emotional disorders.
\end{abstract}

Key words. Depression, anxiety, emotional disorders, self-schema, self-referent encoding task.

Correspondencia: Prof. A. Ruipérez.. Dpto. de Psicologia. Facultad de CC Sociales y Humanas. Universitat Jaume I. Campus de Borriol. 12080-Castellón. España. E-mail: ruiperez@psb.uji.es. 


\section{INTRODUCCIÓN}

Durante las dos últimas décadas el estudio psicológico de los trastornos emocionales y del estado de ánimo ha estado ligado de una manera crucial a la perspectiva cognitiva. Desde este enfoque se han generado diversos modelos explicativos, cada uno de los cuales enfatiza el papel de algún grupo especial de variables cognitivas y/o comportamentales para explicar la génesis y/o el mantenimiento de estos trastornos.

Uno de los modelos más influyentes es sin duda el propuesto por Beck (Beck, 1976, 1987, 1991; Beck y Emery, 1985; Beck, Rush, Shaw y Emery, 1979). Y el constructo psicológico de "esquema" ocupa un lugar central en este modelo. En términos generales, un esquema puede ser concebido como una estructura de la memoria que contiene un conjunto organizado de conocimientos, o sea de información, que interactúa activamente con la información entrante, a través de procesos tales como la dirección selectiva de la atención hacia aquellos elementos que son congruentes con la información almacenada, o dirigiendo los procesos de búsqueda y comparación con esa información. Por lo tanto, los esquemas no solo son estructuras organizadas de datos, sino que también ejercen un papel activo en la selección, la adquisición, la organización, el almacenamiento y la recuperación de nueva información (Williams, Watts, MacLeod y Mathews, 1988).

Un tipo especialmente importante de esquemas son los autoesquemas, que contienen información sobre el sí-mismo. Desde el marco de la teoría de Beck, los autoesquemas de las personas deprimidas contienen generalizaciones de características predominantemente negativas sobre el sí-mismo, que se han ido elaborando a partir de experiencias vitales de pérdida y fracaso (Beck, 1987). De hecho, la expresión de ideas y pensamientos negativos acerca de uno mismo constituye uno de los elementos más característicos de la depresión, desde el punto de vista de las experiencias que relata el propio paciente. Los autoesquemas depresivos pueden ser concebidos como una especie de "base de datos" sobre la que los individuos construyen su identidad, cuya marca de contraste caracteristica es la negatividad. Además, y siempre según el modelo de Beck, se hallan activados de un modo casi permanente en la depresión, lo que entre otras cosas facilita no solo la visión negativa de uno mismo aqui y ahora, sino además la interpretación predominantemente negativa de las propias experiencias (pasadas, presentes y futuras), asi como la recuperación de información negativa, congruente con el contenido nuclear del autoesquema.

En definitiva, según la formulación de Beck, los autoesquemas depresivos constituyen una red muy organizada de información personal predominantemente negativa, además de un conjunto de reglas para evaluar las propias capacidades y valores personales (Segal y Swallow, 1994). Conceptualmente, pueden equipararse a rasgos de personalidad, lo que significa que son consistentes a lo largo de las situaciones y predisponen a ciertos estados episódicos de pensamiento depresivo. Además, se supone que los autoesquemas afectan, como esquemas que son, a la eficacia en el procesamiento de la información que entra en el sistema, de manera que los estímulos altamente autodescriptivos se procesan más rápidamente que los que no tienen esa cualidad (Markus, 1977; Kuiper y Rogers, 1979; Muller, 1982).

La estrategia experimental por excelencia para valorar la existencia de autoesquemas negativos en la depresión es la denominada "Tarea de codificación autorreferente "(TCA) (Self-referent enconding task) (Segal y Swallow, 1994). Se trata de la adaptación de un modelo de laboratorio, inicialmente desarrollado por Craik y Tulving (1975) para poner a prueba el modelo de "niveles de procesamiento" que propusieron Craik y Lockhart (1972) para explicar el funcionamiento de la memoria. Al individuo se le presentan una serie de adjetivos-rasgo con diferentes grados de positividad y negatividad y se le pide que decida en términos categóricos (sí/no) si el rasgo le describe o no. Una vez ha finalizado esta tarea de autoadscripción, se suele administrar una prueba de recuerdo incidental. De este modo, la TCA proporciona diversas medidas que reflejan el funcionamiento de los esquemas subyacentes (Segal y Swallow, 1994).

En primer lugar, el número de palabras positivas y negativas que el individuo ha esco- 
gido como auto-descriptivas, proporciona un indice de la tasa relativa de información positiva y negativa sobre el si-mismo a la que el sujeto tiene mayor accesibilidad. Las investigaciones al respecto coinciden en señalar que los deprimidos se autoadscriben más rasgos negativos que los controles no deprimidos, quienes presentan el patrón inverso de autoadscripción, es decir, escogen más rasgos positivos que negativos como autodescriptivos (MacDonald y Kuiper, 1984; Sanz, 1992, 1994).

En segundo término, el tiempo que la persona tarda en decidir si un rasgo le describe o no, constituye un indicador acerca del contenido de los autoesquemas: si desde el punto de vista teórico, los esquemas facilitan el procesamiento de información cuyo contenido es congruente con la almacenada en el esquema, entonces es de suponer que la persona tardará menos tiempo en decidir (i.e., autoadscribirse) acerca de aquellos rasgos cuyo contenido sea congruente con el de su autoesquema. Algunos estudios han mostrado que las personas no deprimidas presentan menores latencias de respuesta para los rasgos positivos (Kuiper y MacDonald, 1982), mientras que los deprimidos presentan el patrón contrario puesto que tardan menos tiempo en seleccionar rasgos negativos como autodescriptivos (MacDonald y Kuiper, 1984).

La tercera de las medidas tiene que ver con el recuerdo incidental y con el modelo de niveles de procesamiento: se supone que la información cuyo contenido sea congruente con la almacenada en el autoesquema se procesará a un nivel más profundo y que, por lo tanto, se recordará mejor. También en este caso, se han encontrado datos a favor que muestran cómo después de la realización de la TCA los deprimidos recuerdan más rasgos negativos, mientras que los no deprimidos recuerdan más rasgos positivos (Derry y Kuiper, 1981; Kuiper y MacDonald, 1983).

Se han planteado diversas criticas sobre la definición y la adecuación teórica de estos indices y especialmente del segundo de ellos. Por ejemplo, no está claro porqué uno tiene que tardar menos en decidir que SI es sensible, que en decidir que NO es violento, pues en teoria debe tardar lo mismo. Otro tipo de criticas tiene que ver con las dificultades en controlar los efectos de demanda con este tipo de procedimientos, cuyas intenciones pueden resultar bastante transparentes para los sujetos (Mathews y MacLeod, 1994). A pesar de ello, lo cierto es que la TCA se ha convertido en una prueba de elección para valorar la existencia de un "autoesquema depresivo" (Segal y Swallow, 1994). La mayor parte de los estudios realizados con esta tarea se han basado en muestras no clínicas, o bien han utilizado como controles de los deprimidos a personas mentalmente estables, lo que plantea dudas sobre la generalizabilidad de los resultados $y$, sobre todo, sobre la especificidad de los posibles hallazgos a favor de la teoria. Tampoco se ha tenido en cuenta si los diversos tipos de depresión, establecidos sobre la base de parámetros tales como duración del trastorno y gravedad, presentan el mismo patrón de ejecución.

Teniendo en cuenta estas limitaciones, los objetivos del presente trabajo son, primero, poner a prueba los planteamientos de Beck antes enunciados acerca de la existencia de autoesquemas negativos en pacientes deprimidos con diferentes niveles de gravedad y cronicidad y, segundo, comprobar si dichos esquemas son exclusivos de estos individuos o si, por el contrario, se presentan también en otras psicopatologias emocionales, epecificamente en los trastomos de ansiedad.

\section{MATERIAL Y MÉTODOS Muestra}

La muestra para este estudio se obtuvo de los pacientes que acudian de manera consecutiva a consulta al Centro de Salud Mental de Lliria, adscrito a la red pública sanitaria de la Comunidad Valenciana. La composición sociodemográfica de esta población es predominantemente rural y agraria, y dista unos 25 $\mathrm{Km}$. de la ciudad de Valencia. El número total de pacientes incluídos fue de setenta y ocho, de los cuales 56 eran mujeres y 22 eran varones. La media de edad fue de $\mathbf{4 0}$ años (rango: 15 a 70 años).

Los pacientes fueron distribuidos en tres grupos, sobre la base de su diagnóstico principal en el momento del estudio siguiendo criterios DSM-IV (APA, 1994): 22 sujetos fueron diagnosticados de depresión mayor, episodio único (grupo DM), 17 cumplieron crite- 
rios para un diagnóstico de distimia (grupo $\mathrm{Di}$ ), y 39 recibieron un diagnóstico de ansiedad generalizada (grupo $A G$ ). En ningún caso de los pacientes que fueron incluidos en el estudio se plantearon dudas acerca de la coexistencia de más de un diagnóstico principal en el Eje I. Los diagnósticos fueron realizados de manera independiente por el psiquiatra y la psicólogo clínico del Centro, obteniéndose un indice de acuerdo del $95 \%$.

Los otros criterios de inclusión fueron los siguientes: que el paciente diera su consentimiento, que nunca hubiera presentado ideas delirantes ni alucinaciones, que tuviera buena capacidad lectora, no padecer problemas de alcoholismo, adicción a otras drogas o enfermedad orgánica grave y no consumir ningún tipo de psicofármacos en el momento del diagnóstico. Este último aspecto requiere una aclaración adicional: la posible prescripción de psicofármacos una vez realizado el diagnóstico no se consideró criterio de exclusión, ya que los sujetos fueron testados al dia siguiente de ser diagnosticados, en cuyo caso podian llevar como máximo 24 horas de consumo, lo que dificilmente podia afectar a la realización de las pruebas.

\section{Instrumentos}

\section{a) Datos socio-demográficos y de historia clinica}

Todos los pacientes fueron entrevistados según un formato estándar en el que se recogian datos acerca de la historia personal y familiar relacionadas con la alteración y con otras posibles alteraciones mentales previas y su tratamiento. Las variables que se recogieron fueron las siguientes: gravedad de la crisis o episodio actual, antecedentes psicopatologicos personales y familiares, fármacos prescritos para la crisis actual, y tipo de tratamiento y respuesta al mismo en episodios anteriores. Además se recogió información acerca de las características sociodemográficas de cada uno de los pacientes (edad, sexo, estado civil, nivel de instrucción y situación laboral).

\section{b) Instrumentos psicométricos}

Todos los sujetos cumplimentaron las versiones adaptadas a la población española de los siguientes cuestionarios: Inventario de De- presión de Beck (BDI) (Beck, Ward, Mendelson, Mock y Erbaugh, 1961; Conde, Esteban y Useros, 1976), Inventario de pensamientos automáticos negativos (ATQ) (Hollon y Kendall, 1980; Belloch, 1990), Escala de autoconciencia revisada (SCS) (Scheier y Carver (1985); Baños, Belloch y Perpiña, 1990), Inventario de ansiedad estado-rasgo (STAI-E/R) (Spielberger, Gorsuch y Lushene, 1970; Seisdedos, 1988)

\section{c) Tarea de codificación autorreferente (TCA)}

Como ya se indicó en la introducción, la TCA consiste en presentar secuencialmente al sujeto una serie de adjetivos descriptivos (rasgos de personalidad) y pedirle que responda si le describen o no en el momento del experimento. Una vez finalizada la tarea, se le pide que intente recordar tantas cuantas palabras le sea posible.

La TCA se aplicó utilizando un ordenar personal compatible (PC 286), en cuya pantalla iban apareciendo, uno por uno, los estimulos. El orden de presentación de los estimulos fue aleatorio para cada sujeto. El tiempo de exposición no tenía límite, apareciendo una nueva palabra estímulo en el momento que el sujeto dejaba de pulsar la tecla "Sl" o "NO". Se utilizó un teclado expandido, y para facilitar la tarea el "SI" se situó en la barra espaciadora del bloque alfanumérico, y el "NO" en las teclas de dirección, en el bloque de control del teclado. Los sujetos zurdos podian tener la disposición contraria, si bien en nuestra muestra no apareció ningún sujeto con estas caracteristicas. Las teclas fueron recubiertas con etiquetas en forma de casulla donde se marcaba gráficamente el SI y el NO. De esta forma el sujeto apoyaba sus manos en la mesa, ya que las teclas marcadas se situan en la parte inferior más externa del teclado, y además ofrecian una superficie de pulsación más extensa que cualquier otra tecla. También se asoció un estímulo discriminativo auditivo cuando el sujeto no dejaba de presionar la tecla una vez que ya habia respondido.

El material utilizado consistió en 75 adjetivos que describieran rasgos de personalidad con valencia emocional positiva, negativa, o neutra. Asi, del total de rasgos escogidos, 25 tenian un contenido relacionado con estados 
de ánimo negativos ("adjetivos negativos"), 25 con estado de ánimo positivos ("adjetivos positivos") y 25 eran neutros en relación a estado de ánimo ("adjetivos neutros"). Los estímulos se presentaron en forma masculina o forma femenina, según fuera el género del paciente.

La selección de los estímulos se realizó en dos fases. La primera de ellas consistió en revisar las investigaciones publicadas hasta el momento de inicio del estudio en las que se hubieran utilizado estímulos-rasgo con contenido depresivo, ansioso o neutro (por ejemplo, Bradley y Mathews, 1983; Greenberg y Alloy, 1989; Ingram, Kendall, Smith, Donnell y Ronan, 1987; Klieger y Cordner, 1990; Kuiper, Derry y MacDonald, 1982; Myers, 1984; Sanz, 1992; Vázquez y Alloy,1987).

Una vez elaborada la lista inicial con 200 palabras, el paso siguiente consistió en pedir a 10 jueces (psicólogos clínicos y psiquiatras experimentados) que evaluaran en una escala de 0 (no adecuado) a 10 (muy adecuado), la adecuación de los rasgos seleccionados para describir características personales con valencia emocional negativa ("rasgos depresivos), positiva ("rasgos positivos"), o neutra ("rasgos neutros"). Todos los rasgos fueron evaluados según cada una de las tres categorias. La selección final se basó en los rasgos que hubieran obtenido una puntuación media $\leq$ a 6 para una sola de las categorias y una puntuación $\geq$ a 3 en las otras dos. La lista definitiva se encuentra en el Apéndice. Finalmente, las autoras seleccionaron además diez sustantivos para que sirvieran como "estímulos ensayo" y permitieran a los sujetos familiarizarse con el procedimiento de la prueba $y$, especialmente, con el uso del teclado. Algunos de estos sustantivos estaban relacionados con comida y otros hacian referencia a objetos cotidianos. Ninguno de ellos se incluyó, lógicamente, en el análisis de los resultados.

\section{Procedimiento}

Todos los sujetos fueron examinados de modo individual. Una vez realizada la entrevista diagnóstica por el psicólogo clínico y/o por el psiquiatra del Centro, y descartados los posibles criterios de exclusión, el paciente era informado sobre la posibilidad de participar voluntariamente en un estudio sobre las caracteristicas de personalidad de la población valenciana. Si daba su consentimiento, se le proporcionaban los instrumentos psicométricos $y$, una vez completados en presencia de una de las autoras (MAR), que en ese momento era la psicólogo clínico del Centro, se le citaba para el día siguiente, siempre que el paciente no hubiera presentado dificultades lectoras yl o de comprensión de los instrumentos psicométricos.

La sesión comenzaba solicitando al paciente que leyera en voz alta un texto breve (250 palabras) que aparecia en la pantalla del ordenador. Dada la procedencia mayoritariamente rural de la muestra, el propósito de este primer paso era re-asegurar de nuevo la capacidad lectora y de comprensión del sujeto, con el fin de evitar posibles interferencias posteriores en la realización de la TCA. Posteriormente se le pedia que resumiera en sus propias palabras el mensaje del texto. Se tomaron como criterios de adecuación la comprobación de la comprensión del mismo, asi como la estimación del tiempo empleado en la lectura y los errores de pronunciación. Una vez superada esta primera fase, se le proporcionaba la explicación pormenorizada de los mecanismos para realizar adecuadamente la TCA. Una vez el paciente habla comprendido las instrucciones, comenzaba a realizar los 10 ensayos de prueba. En el caso de que surgieran dudas, o una mala ejecución, se le volvia a explicar y de nuevo realizaba los ensayos de prueba. Este procedimiento se repitio hasta asegurarnos de que el paciente se habia familiarizado correctamente con la prueba, si bien normalmente bastaba un ensayo para dar comienzo a la TCA propiamente dicha. Una vez finalizada la tarea, se administraba una prueba distractora (el subtest de dígitos del WAIS, en su forma directa) y, a continuación se procedia con la tarea de recuerdo incidental.

\section{Análisis de los datos}

Para la comparación de los sujetos en las variables psicométricas, sociodemográficas y de historia clínica, se utilizaron pruebas paramétricas (ANOVAs) o no paramétricas $\left(\chi^{2}\right)$, según fuera la naturaleza de las variables analizadas. 
En cuanto al análisis de la TCA se aplicaron tres diseños ANOVAs con medidas repetidas para las variable intra-sujetos: el primero, relacionado con la búsqueda de diferencias entre grupos en cuanto al contenido del autoesquema, operacionalizado en términos del tipo (valencia) de rasgos elegidos y no elegidos como autodescriptores, contenia una variable entre-sujetos ( 3 grupos: $\mathrm{DM}, \mathrm{Di}, \mathrm{AG}$ ), y dos variables intra-sujetos: valencia emocional de la autoadscripción (positiva, negativa, neutra) y tipo de autoadscripción (positiva o negativa). El segundo diseño tenia por objeto analizar la accesibilidad del autoesquema, y se mantuvo la misma variable entre-sujetos, pero se variaron las intra-sujetos: tiempo de decisión léxica para cada autoadscripción (positiva, negativa), y tiempo empleado según la valencia de los descriptores (positiva, negativa, neutra). El tercero de los diseños tenia por objeto estudiar las tasas de recuerdo de los adjetivos presentados y su contenido (valencia). La variable entre-grupos fue, de nuevo, la misma que en los dos diseños previos ( 3 grupos), pero en este caso las variables intragrupo fueron la tasa de recuerdo según su valencia (positiva, negativa, neutra) y la tasa de recuerdo de los rasgos que habian sido previamente escogidos como autodescriptivos y como no autodescriptivos.

En todos los casos en los que fue necesario se transformaron las puntuaciones brutas con el fin de igualar las varianzas y/o eliminar los efectos de suelo Para el primero de los diseños, las puntuaciones directas obtenidas se transformaron calculando la proporción del número total de adscripciones posibles para cada sujeto y después se aplicó la raíz cuadrada del arcoseno a cada puntuación (Wine, 1971; Markus y Nurius 1987). En cuanto a los tiempos de decisión léxica, transformamos los valores directos obtenidos en mseg. La transformación que se realizó fue la inversa del tiempo empleado por el sumatorio de los rasgos que el sujeto se adscribia o no en cada categoria. Finalmente, puesto que los datos obtenidos en la prueba de recuerdo mostraron un efecto de "suelo", las puntuaciones brutas fueron transformadas según el siguiente esquema: el $50 \%$ de las puntuaciones directas tomó el valor de 0 , extendiendo el resto de los valores simétricamente hasta llegar a los extremos ( -1 para el $0 \%$ y +1 para el $100 \%$ ). Posteriormente se utilizo el factor de potencia ( 1 menos la pendiente) para conseguir que la escala de valores antes mencionada, no tuviera un carácter lineal sino exponencial, "deformando" simétricamente los valores extremos, es decir, próximos al $0 \%$ y al $100 \%$.

El nivel mínimo de significación estadistica exigido fue $\leq 0,05$. Los análisis fueron realizados con el paquete estadístico SPSS para Windows, versión 6.1.

\section{RESULTADOS}

El análisis de las variables socio-demográficas no arrojó diferencias significativas entre los tres grupos de pacientes en ninguna de las variables contempladas. Por lo que se refiere a los datos relacionados con la historia clínica, se observaron diferencias entre los grupos en cuanto a las variables relacionadas con la prescripción de psicofármacos, en el sentido de que los pacientes con depresión mayor y los ansiosos recibian mayores dosis de fármacos ansioliticos $\left(\chi^{2}=7,77 ; p<0,002\right)$ y antidepresivos $\left(\chi^{2}=20,45 ; p<0,0001\right)$ que los distímicos. Además, los pacientes con depresión mayor fueron calificados como más graves que los distímicos $y$ ansiosos $\left(\chi^{2}=15,94 ; p<0,0001\right)$, no existiendo diferencias en este parámetro entre estos dos últimos grupos.

Las puntuaciones medias de los sujetos en los instrumentos psicométricos, así como las diferencias observadas entre los 3 grupos en cada uno de ellos, se incluyen en la tabla 1. Como puede observarse, solo se obtuvieron diferencias significativas en el Inventario de Beck y en la subescala de autoconciencia pública del cuestionario SCS. En el primer caso, fueron los pacientes con depresión mayor quienes obtuvieron las puntuaciones más elevadas, situándose los distímicos y los ansiosos en segundo y tercer lugar, respectivamente. El análisis post-hóc reveló que la fuente de las diferencias se hallaba precisamente entre el primer grupo y los otros dos. En cuanto a la autoconciencia pública, él patrón de resultados es justamente el contrario: los ansiosos y distímicos obtuvieron puntuaciones más elevadas que los pacientes con depresión mayor. 
Tabla 1.- Datos normativos y diferencias entre grupos diagnósticos en los cuestionarios*

\begin{tabular}{|c|c|c|c|c|c|}
\hline \multicolumn{2}{|c|}{ INSTRUMENTOS } & \multirow{2}{*}{$\begin{array}{c}\begin{array}{c}\text { Depresión } \\
\text { Mayor } \\
(\mathrm{N}=22)\end{array} \\
27,18 \\
(8,95)\end{array}$} & \multirow{2}{*}{$\begin{array}{c}\text { Distimia } \\
\begin{array}{c}(\mathbf{N}=17) \\
23,71 \\
(7,23)\end{array}\end{array}$} & \multirow{2}{*}{$\begin{array}{c}\begin{array}{c}\text { Ansledad } \\
\text { Generallzada } \\
(\mathrm{N}=39)\end{array} \\
\begin{array}{c}18,74 \\
(9,39)\end{array}\end{array}$} & \multirow{2}{*}{$\begin{array}{c}\begin{array}{c}F \\
(p<)\end{array} \\
\begin{array}{c}6,71 \\
(0.002)\end{array}\end{array}$} \\
\hline BDI & & & & & \\
\hline & PÚBLICA & $\begin{array}{l}15,64 \\
(3,61)\end{array}$ & $\begin{array}{l}18,29 \\
(3,29)\end{array}$ & $\begin{array}{l}18,59 \\
(4,13)\end{array}$ & $\begin{array}{c}4,75 \\
(0,01)\end{array}$ \\
\hline \multirow[t]{2}{*}{ scs } & PRIVADA & $\begin{array}{l}23,36 \\
(4,80)\end{array}$ & $\begin{array}{l}23,71 \\
(4,01)\end{array}$ & $\begin{array}{l}24,85 \\
(4,83)\end{array}$ & $\begin{array}{l}0,82 \\
\text { (NS) }\end{array}$ \\
\hline & A. SOCIAL & $\begin{array}{l}15,36 \\
(5,32)\end{array}$ & $\begin{array}{l}15,00 \\
(4,56)\end{array}$ & $\begin{array}{l}14,95 \\
(3,39)\end{array}$ & $\begin{array}{l}0,07 \\
\text { (NS) }\end{array}$ \\
\hline ATQ & & $\begin{array}{c}70,95 \\
(24,78)\end{array}$ & $\begin{array}{c}68,65 \\
(22,74)\end{array}$ & $\begin{array}{c}62,67 \\
(17,73)\end{array}$ & $\begin{array}{l}1,22 \\
\text { (NS) }\end{array}$ \\
\hline STAI-E & & $\begin{array}{c}31,23 \\
(10,19)\end{array}$ & $\begin{array}{c}27,76 \\
(12,75)\end{array}$ & $\begin{array}{c}27,54 \\
(13,04)\end{array}$ & $\begin{array}{l}0,65 \\
\text { (NS) }\end{array}$ \\
\hline STAI-R & & $\begin{array}{c}41,41 \\
(10,48)\end{array}$ & $\begin{array}{c}41,59 \\
(10,97)\end{array}$ & $\begin{array}{l}38,92 \\
(9,00)\end{array}$ & $\begin{array}{l}0,69 \\
\text { (NS) }\end{array}$ \\
\hline
\end{tabular}

* Los datos se representan como Media (Desviación Estándar).

BDI = Inventario para la Depresión de Beck; SCS = Escala de Autoconciencia; $\mathbf{A T Q}=$ Cuestionario de Pensamientos Automáticos Negativos; STAI-E = Inventario de Spielberger para la Ansiedad-Estado; STAI-R = Inventario de Spielberger para la Ansiedad-Rasgo.

NS $=$ no significativa estadisticamente

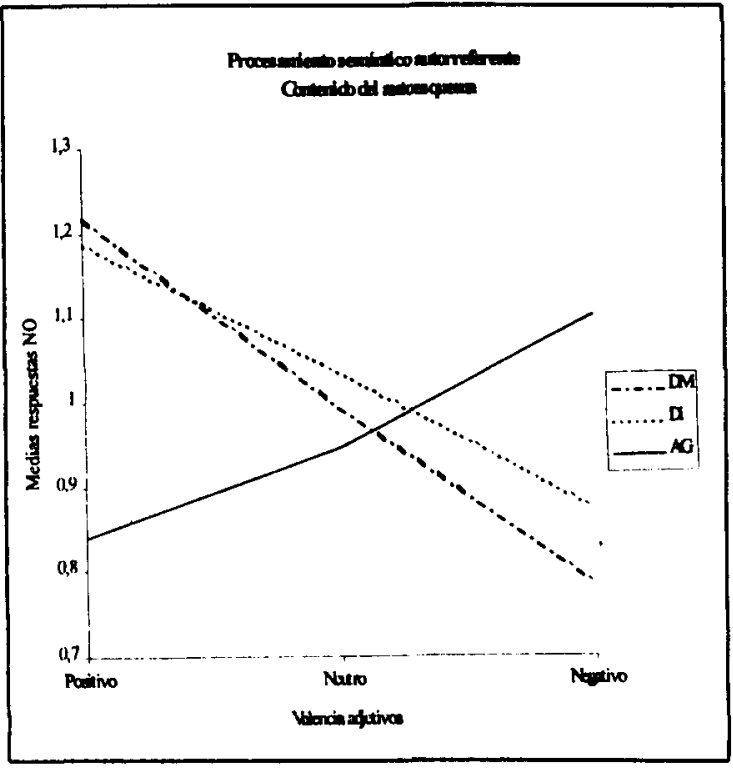

Figura 1.- Media de respuestas "No me describe"

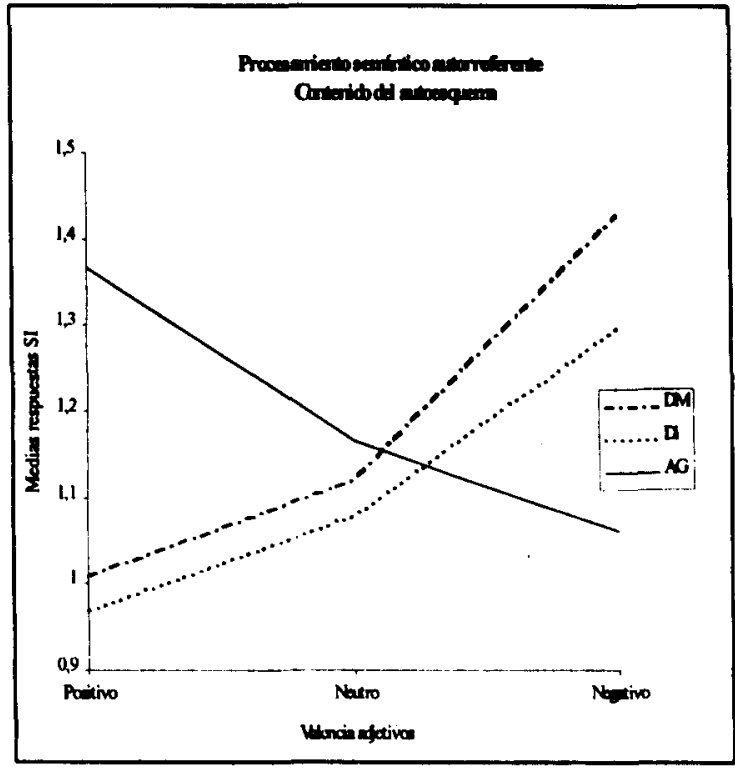

Figura 2.- Media de respuestas "Si me describe" 
Por lo que se refiere a los resultados obtenidos con la TCA en relación con el contenido del autoesquema, encontramos diferencias significativas en cuanto a la valencia de los rasgos elegidos como autodescriptivos $(F=4,15 ; p<0,001)$, asi como efectos significativos de la interacción grupo diagnóstico $x$ valencia de los rasgos elegidos como autodescriptivos $(F=3,94 ; p<0,001)$. Los análisis posthoc revelaron que los pacientes con de-presión mayor y los distímicos se autoadscri-bian más rasgos negativos que los ansiosos ( $F=$ $3,16 ; p<0,007)$, además de rechazar un mayor número de rasgos positivos como elementos de autodescripción $(F=4,34 ; p<0,01)$. No hubo diferencias entre los tres grupos en cuanto a los rasgos neutros elegidos o rechazados como autodescriptivos. Las figuras 1 y 2 ejemplifican estos resultados.

En cuanto a la accesibilidad del autoesquema, hubo diferencias entre los tres grupos en cuanto al tiempo de decisión léxica ( $F=5,63$; $p<0,005)$, pero no encontramos efectos significativos relacionados con la valencia $(F=1,35)$ ni en la interacción grupo $x$ valencia $(F=0,87)$. Los análisis post-hoc mostraron que las diferencias entre grupos se debian al hecho de que el grupo de pacientes con depresión mayor empleaba más tiempo en sus decisiones, independientemente del contenido o valencia de los rasgos sobre los que debla decidir acerca de su capacidad como autodescriptores. Dicho en otros términos, parecían mostrar una lentitud generalizada de respuesta. Por su parte el grupo de pacientes distímicos tardaba menos tiempo que los otros dos grupos de pacientes en todos los casos, excepto en la decisión sobre los rasgos positivos con los que no se identificaban. Las figuras 3 y 4 resumen estos resultados.

Finalmente, el análisis realizado sobre el contenido del material recordado por los pacientes, reveló que no existian diferencias estadisticamente significativas entre los grupos en cuento al número de adjetivos recordados $(F=2.32 ; p<0.10)$. Tampoco hubo diferencias significativas en la interacción grupo $x$ por valencia $(F=1.99 ; p<0.09)$. Sin embargo, si detectamos diferencias estadísticamente significativas en cuanto a las características de los adjetivos que recordaban ( $F=14.38$; $\mathrm{p}<0.00)$. Los análisis post-hoc arrojaron los siguientes resultados (véanse las figuras 5 y 6):

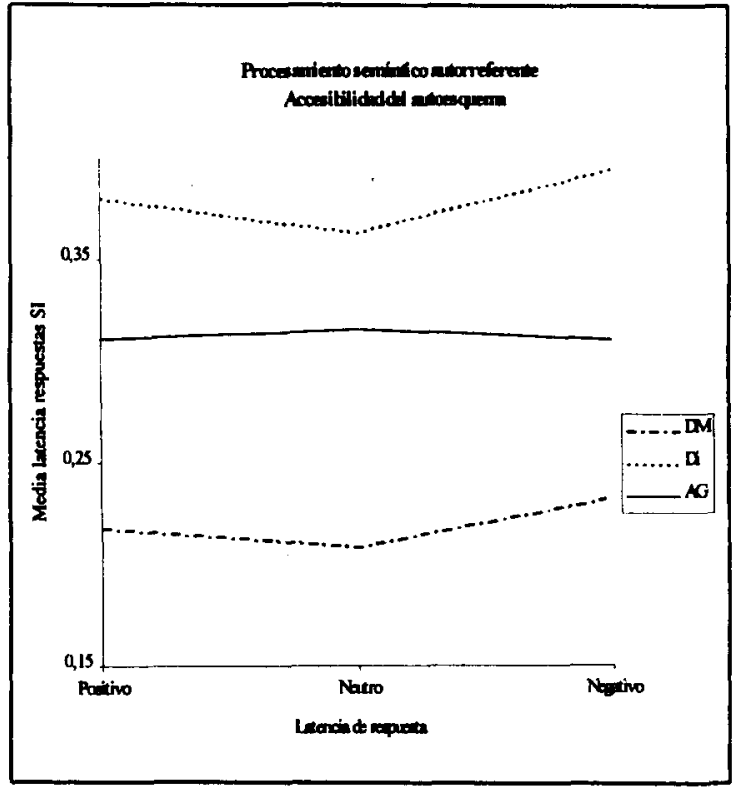

Figura 3.- Latencias de respuesta para "Si me describe"

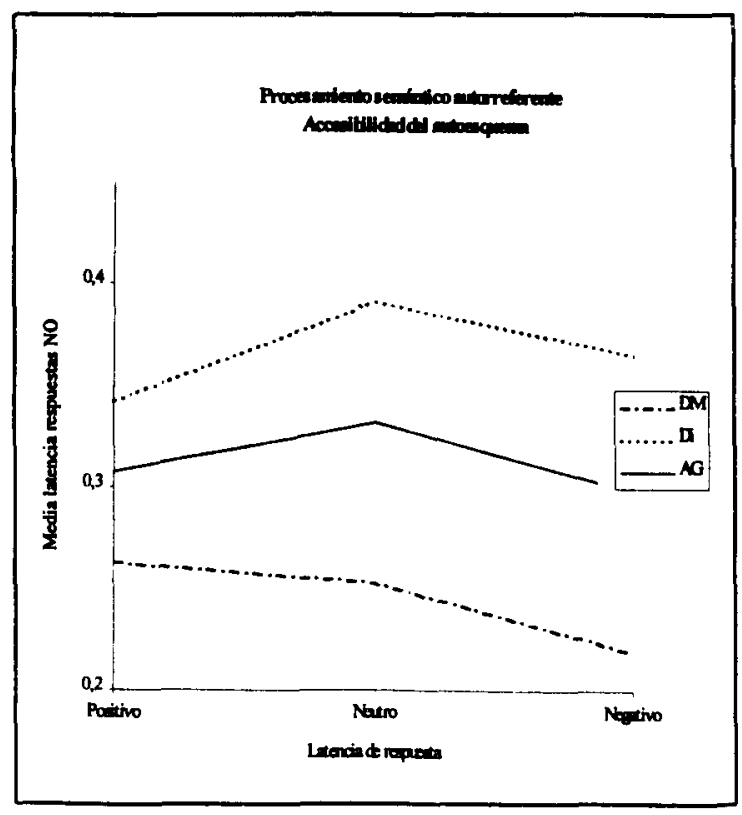

Figura 4.- Latencias de respuesta para "No me describe"

a) Los tres grupos diferian en cuanto al número de rasgos negativos que recordaban $(F=4.14 ; p<0.01)$. Este efecto se debia al hecho de que los DM recordaban más rasgos negativos que los otros dos grupos.

b) No hubo diferencias en cuanto al número de rasgos positivos recordados por los tres grupos ( $F=0.39 ; p<0.67)$. 


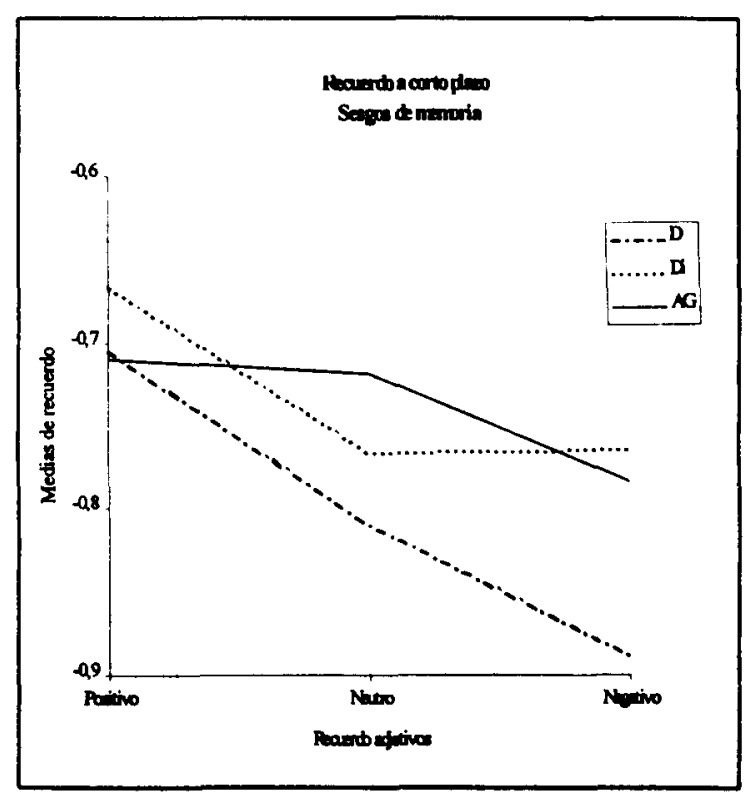

Figura 5.- Contenido de los adjetivos que se recuerdan

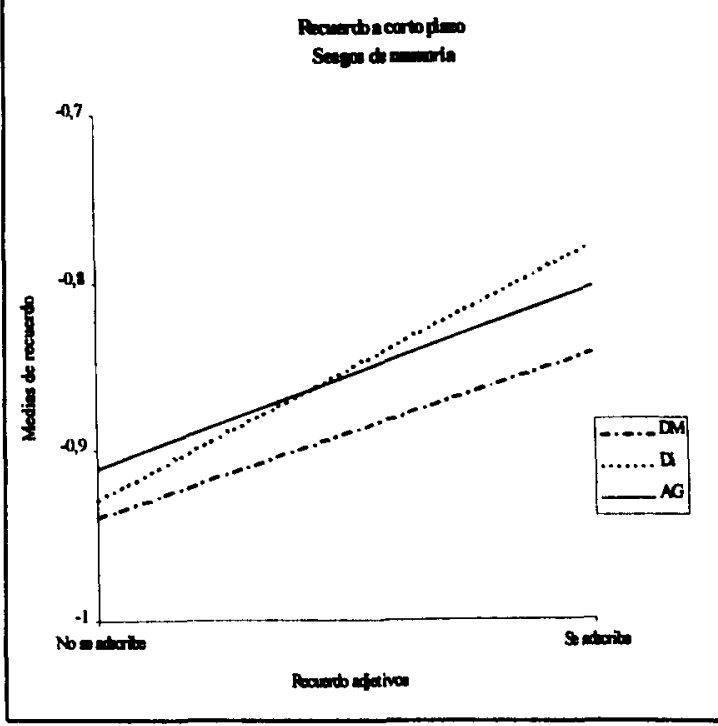

Figura 6.- Recuerdo de los rasgos elegidos $y$ no elegidos como autodescriptivos

c) Hubo diferencias en cuanto al número de rasgos neutros recordados por cada grupo ( $F=3.02 ; p<0.05)$, debido a que los $D M$ recordaban más adjetivos neutros que los AG.

Asi pues, los Depresivos mayores presentaron mayores tasas de recuerdo de adjetivos negativos que los otros dos grupos de pacien- tes, no existiendo a su vez diferencias en este aspecto entre ambos grupos.

Por lo que se refiere a la cuestión de si la calidad del recuerdo se haya asociado al hecho de que hubiera habido previamente autoadscripción, independientemente de la valencia de los elementos a recordar se realizo un ANOVA 3 (grupos) $\times 2$ (me describe/no me describe). Los resultados mostraron que no existian diferencias estadisticamente significativas entre los grupos en cuanto a la forma de proceder de cada uno de ellos para cada uno de los niveles de la variable dependiente, $(F=2,52$; $p<0.08)$; tampoco se encontraron diferencias significativas en la interacción grupo $x$ variable, $(F=2,07 ; p<0.13)$. Pero sí se encontraron diferencias estadisticamente significativas en cuanto a si los adjetivos que eran más recordados, habian sido previamente considerados como autorreferentes o no $(F=129.42 ; p<0.00)$. Los resultados muestran que todos los grupos presentaban mejor recuerdo de aquellos adjetivos que hablan elegido para audescribirse, fuera cual fuese la valencia de los mismos. No hallamos diferencias significativas entre los grupos en los análisis post-hoc realizados. En consecuencia, los resultados del análisis previo quedan obviamente matizados con este segundo análisis: los deprimidos mayores recuerdan, efectivamente, mayor número de rasgos negativos escogidos como autodescriptivos que los otros grupos, sencillamente porque se autoadscribieron, ya de entrada, un mayor número de ellos.

\section{DISCUSIÓN}

Investigaciones anteriores en este campo han mostrado que la tarea de codificación autorreferente, es sensible a la depresión y que, por lo tanto, permite distinguir fiablemente entre grupos de individuos deprimidos y no deprimidos, siempre que el contenido de las palabras que se utilicen sea el apropiado. En esta tarea, como ya hemos descrito, se presentan visualmente adjetivos-rasgos de personalidad preseleccionados, y se pide a los sujetos que decidan (categorialmente: si/no) si la palabra en cuestión les parece auto-descriptiva; además de la respuesta (decisión), se mide el tiempo requerido para la misma. Este tipo de tareas incluye también una prueba de 
recuerdo incidental de los adjetivos presentados, por lo que este paradigma toma en consideración tres elementos: a) el patrón de autoadjudicación de adjetivos-rasgo de contenido emocional; b) el tiempo de latencia que emplea el individuo para adoptar una decisión; $y$, c) la tasa de recuerdo de los adjetivos-rasgo que fueron señalados (codificádos) como autorreferentes. Comentaremos por separado los resultados obtenidos para cada uno de tales parámetros.

Por lo que se refiere al primer aspecto, esto es, el tipo de rasgos (positivos versus negativos) que cada uno de los tres grupos de pacientes escogió como autodescriptivos, nuestros resultados señalaban que los pacientes con un diagnóstico de depresión, ya fuera distimia o depresion mayor, escogían un mayor número de rasgos negativos que de positivos y neutros; paralelamente, este patrón de elección era el inverso al encontrado en los pacientes con ansiedad generalizada. Estos resultados son coincidentes con la mayor parte de la literatura al respecto. Davis (1979a, 1979b) fue el primero que aplico el paradigma TCA a la depresión. Planteaba que los sujetos deprimidos estimaban más adjetivos negativos como autodescriptivos y que esta tendencia más fuerte a adoptar adjetivos negativos estaba asociada a estados depresivos más largos (Davis y Unruh, 1981).

En un estudio mucho más reciente sobre memoria, depresión y autoesquema (Brewin, Smith, Power y Furnham, 1992), realizado con pacientes depresivos y un grupo control de sujetos normales, se encontró que los sujetos deprimidos se auto-adscribian más adjetivos negativos cuando se les pedia que respondiesen según se veían a si mismos en ese momento que cuando se les pedia que realizaran la misma valoración pero con respecto a otro momento de su vida y/o de ellos mismos en general. En estos dos últimos casos, los pacientes seleccionaban por igual adjetivos negativos y positivos para describirse. Sin embargo los sujetos no deprimidos se describian siempre con más términos positivos. Este tipo de resultados ponen de relieve que la depresión actúa como una especie de amplificador de los propios defectos, intensificando la visión negativa de uno mismo y/o disminuyendo la autopercepción negativa, en el sentido si- guiente: el estado depresivo incita al individuo a valorarse negativamente en comparación con su si-mismo habitual o normal (i.e., no depresivo), es decir, que la depresión activa procesos de comparación con un sí-mismo estándar anterior $y$, probablemente, futuro.

En consonancia con esta argumentación, Vázquez y Alloy (1987) aportan evidencia respecto a que los individuos normales poseen un autoesquema más positivo.. En tareas de autoadscripción de rasgos los sujetos normales dieron más autodescripciones extremas, es decir, señalaron todos los adjetivos positivos y rechazaron todos los negativos en mayor porcentaje que los grupos de ansiosos o depresivos con quienes se les comparaba. Asi pues estas personas no deprimidas ni ansiosas, parecen caracterizarse por un autoesquema de contenido muy positivo. Este dato resulta consistente con otra serie de investigaciones bien documentadas sobre la existencia de sesgos cognitivos optimistas por parte de individuos no deprimidos (Abramson y Alloy, 1981; Alloy y Abramson, 1988; Lewinsohn, Mischel, Chaplain y Barton, 1980). En nuestro caso ese autoesquema positivo sería también caracteristico de los pacientes con un diagnóstico de ansiedad generalizada, quienes se parecerían a las personas normales de los citados estudios, puesto que se adscriben significativamente un mayor número de adjetivos positivos que negativos. En definitiva, y por lo que se refiere a la variable que estamos considerando, nuestros resultados apoyan la hipótesis general que postula la existencia de un autoesquema con contenido negativo en los deprimidos que, además, sería relativamente especifico de estos pacientes frente a los ansiosos.

Por lo que se refiere al segundo parámetro, esto es la latencia de respuesta o tiempo empleado por los sujetos para decidir acerca de si un rasgo les describe o no, ya hemos dicho que se ha venido considerando como un indicador del grado de accesibilidad del autoesquema. En este sentido, el grupo de Kuiper (Kuiper, MacDonald y Derry, 1983; Kuiper y Olinger, 1986; Kuiper Olinger, MacDonald y Shaw, 1985), ha argumentado que los tiempos de decisión más rápidos implican un procesamiento más eficiente y un esquema cognitivo más fuerte. Nuestros resultados acerca de una mayor lentitud en todos los casos de los pa- 
cientes con depresión mayor apuntarian, según lo anterior, a una menor accesibilidad del autoesquema, que además es independiente de su contenido o dirección (positiva o negativa), mientras que la mayor rapidez de respuesta, en todos los casos, de los pacientes Distimicos indicaria una mayor accesibilidad del autoesquema en este grupo. Finalmente, los ansiosos se hallarian en un punto intermedio entre los dos grupos de deprimidos. La mayor lentitud general de los DM en la tarea es, por otro lado, compatible con otros estudios que indican que el estado de ánimo deprimido tiende a relacionarse con una peor ejecución en tareas que involucran la valoración del tiempo empleado para realizarlas, que es independiente del contenido de los estímulos sobre los que se realiza la decisión, y que se explicaría apelando a una disminución general de los recursos atencionales conscientes que están disponibles para la realización de la tarea (cfr. Ellis y Ashbrook, 1988; Ellis, Thomas y Rodriguez, 1984).

Por otra parte, nuestros resultados no apoyan la existencia de un efecto selectivo del estado de ánimo deprimido sobre la percepción de estímulos congruentes con dicho estado, tal y como se postula desde la teoría de red asociativa de Bower (1981), puesto que desde dicha teoría, los deprimidos percibirian antes las palabras "depresivas" lo que incidiría en su rapidez a la hora de tomar una decisión sobre su potencial capacidad para describirles. Este resultado es congruente con los procedentes de otras investigaciones realizadas con pacientes y con individuos a los que se les ha inducido un estado de ánimo negativo (Clark, Teasdale, Broadbent y Martin, 1983; Challis y Krane, 1988; MacLeod, Tata y Mathews, 1987), asi como los realizados con sujetos subclínicamente deprimidos (Gotlib, McLachlan y Katz 1988; Hill y Dutton, 1989; Ruiz-Caballero y Bermúdez, 1993).

Sin embargo hay que destacar que en los pacientes con diagnóstico de Distimia sí que se produjo el efecto de un menor tiempo en la autoadscripción de rasgos negativos frente a los otros dos grupos de pacientes. A primera vista este resultado vendria a apoyar las hipotesis formuladas en este sentido; pero hay que tener en cuenta que, a nivel intra-grupo, no hay diferencias en cuanto al tiempo que tar- dan los Di para decidir sobre la autoadscripción de rasgos en función de la valencia de los mismos. Es posible que el bajo nivel de instrucción de los pacientes de nuestra muestra haya ejercido un efecto perturbador en esta tarea. Algunas formulaciones teóricas sobre el reconocimiento (por ejemplo, Foster, 1978), han sugerido que la identificación semántica de una palabra es posterior a su identificación léxica y, en consecuencia, el valor o tono emocional de la palabra no influiria en el tiempo de decisión léxica. Desde este planteamiento, cuanto más desconocida o poco habitual sea la palabra-estímulo para un individuo, menos influirá su valencia emocional a la hora de decidir sobre la misma. Si esto es asi, es posible que, como acabamos de argumentar, el escaso nivel de formación intelectual y hábito lector de nuestros pacientes haya ejercido un efecto perturbador sobre la ejecución correcta de la tarea propuesta. En todo caso y a pesar de las consideraciones anteriores, parece que lo más correcto es concluir que las hipótesis sobre una mayor accesibilidad del autoesquema negativo en los pacientes deprimidos no se han visto avaladas por nuestros resultados.

Por lo que se refiere al tercero de los parámetros consideradas, el recuerdo, la teoria asociativa del afecto sugiere que la información que es congruente con el estado de ánimo, es procesada (codificada) mejor y más eficientemente, lo que facilita su recuperación, en comparación con aquella información que no es congruente con el estado de ánimo. Además, se postula que este efecto de congruencia entre codificación y recuerdo será mayor o más importante si el material estimular que se va a utilizar en el proceso de elaboración / codificación guarda relación con el propio individuo (material auto-referente) o se fomenta la utilización de material específico, como por ejemplo situaciones o personajes concretos.

Uno de los primeros trabajos publicados en esta línea de investigación utilizando pacientes deprimidos fue el de Derry y Kuiper (1981). Utilizaron adjetivos de contenido depresivo y de contenido no depresivo, y sus resultados revelaron un mejor recuerdo para aquellos adjetivos que eran juzgados como autorreferentes (lo que era de esperar, según el paradigma de los niveles de procesamiento), y cuyo conte- 
nido se correspondía con el estado emocional del sujeto (según se postula en la teoria de Bower).

La literatura publicada al respecto en los últimos años ha sido objeto de varias revisiones importantes, entre las que cabe destacar las de Blaney (1986), Brewin (1988), Riskind (1989), Singer y Salovey (1988), o Williams, Watts, MacLeod y Mathews (1988). La conclusión compartida entre estos autores es la de que el efecto de congruencia puede detectarse en algunas situaciones con bastante claridad, mientras que en otros casos resulta cuanto menos poco consistente. Por ejemplo, en la revisión de Blaney se concluye que el efecto de congruencia con el estado de ánimo se detecta en pacientes deprimidos solo si el material estimular tiene contenido autorreferente. Sin embargo, en la depresión subclínica y en situaciones de inducción experimental de estados de ánimo, los resultados son poco concluyentes y en general, inconsistentes. Por su parte, Singer y Salovey (1988) señalan que el efecto de congruencia es bastante robusto si se examina la codificación, pero que es menor cuando se tiene en cuenta el momento de la recuperación en situaciones de ánimo inducido $y$, finalmente, que es inconsistente cuando se estudia en sujetos clínica o subclínicamente deprimidos.

Matt, Vázquez y Campbell (1992) han realizado un metaanálisis de la literatura sobre este tema y, entre otras cosas, concluyen que: a) los quince estudios revisados, realizados con individuos no deprimidos aportan datos a favor de la existencia de un sesgo mnésico relacionado con el material cuya valencia afectiva es positiva; este efecto es importante y no presenta variaciones substanciales en función de las características metodológicas de los diversos estudios; b) cuando se utiliza población subclínicamente deprimida, los resultados más consistentes apuntan a que no existe el efecto de congruencia. Esta conclusión se basa en el análisis de siete estudios publicados; $y, c)$ cuando las muestras de referencia utilizadas son de pacientes con depresión clínica, fundamentalmente con diagnóstico de depresión mayor, y puntuaciones medias de 24 en el BDI, los efectos de congruencia que se registran son importantes y van en la dirección opuesta a los hallados para sujetos normales: los deprimidos recuerdan mejor el material con valencia afectiva negativa, el cual es congruente con su estado de ánimo. En este grupo se incluyeron siete estudios, de los que cuatro utilizaban, como en el que aqui presentamos, material autorreferente (Bradley y Mathews, 1983, 1988; Derry y Kuiper, 1981; Roth y Rhem, 1980), y a excepción del primero de ellos, los otros tres utilizaron aprendizaje incidental, como en nuestro caso.

Nuestros resultados indicaron que los pacientes con depresión mayor recordaban, como se habia postulado y como se deduce de la literatura comentada, más adjetivos negativos que los otros dos grupos. Ahora bien: este efecto podia deberse al hecho de que, previamente, los pacientes se habian autoadscrito un mayor número de tales adjetivos. Sin embargo, a la luz de la primera conclusión que antes comentamos sobre los estudios revisados por Matt et al. (1992), así como según los planteamientos de otros autores (Ferguson, Rule y Carlson, 1983; Keenan y Baillet, 1980; Kuiper y Rogers, 1979; McCaul y Maki, 1984), este último aspecto no sería relevante, dado que se ha constatado que el efecto de autorreferencia, o sea, la superioridad mnésica que se produce cuando el material es procesado con referencia al si-mismo, es independiente de si los adjetivos-estímulo son autodescriptivos o no, esto es, si los sujetos contestan "si" o "no" a la instrucción de autorreferencia. Por otro lado, si el efecto mencionado fuera el más relevante, hubiera debido producirse el mismo patrón de resultados en los distímicos, puesto que inicialmente se hablan autoadscrito más rasgos negativos. Sin embargo, estos pacientes no recordaban más rasgos negativos, a pesar de que previamente se los hubieran autoadscrito.

Finalmente queremos resaltar el hecho de que la diferente ejecución mostrada por los pacientes con depresión mayor y los distímicos, excepción hecha de la tendencia similar a autoadscribirse más rasgos negativos que positivos o neutros, apoya la necesidad de establecer diferencias entre ambos tipos de trastornos del estado de ánimo, al menos tal y como se formulan en el Manual DSM-IV. Estas diferencias deberían ser tenidas en cuenta cuando se disefian investigaciones acerca de las características cognitivas de los pacientes 
deprimidos. Por otro lado, la no existencia de diferencias notables entre los pacientes distímicos y los ansiosos, plantea dudas acerca de la idoneidad de considerar ambas entidades como radicalmente diferentes, al menos desde el punto de vista de su rendimiento en tareas como las realizadas en este estudio. En el mejor de los casos, estos resultados indicarian la necesidad de replantear los criterios diagnósticos de la ansiedad generalizada, de la distimia, o de ambas. Aunque parece claro que el miedo y la tristeza constituyen dos estados emocionales diferenciados, tanto en la clínica como desde el punto de vista psicopatológico, la distinción entre ansiedad generalizada y depresión crónica, constituye un problema que hoy por hoy no parece estar resuelto.

\section{REFERENCIAS BIBLIOGRÁFICAS}

Abramson, L.Y. y Alloy, L.B. (1981). Depression, non-depression and cognitive illusions: Replay to Schwart. Joumal of experimental Psychology. General, 100, 436-447.

Alloy, L.B. y Abramson, L.Y. (1988). Depressive realism: four theoretical perspectives. En L.B. Alloy (Ed.), Cognitive Processes in Depression, págs. 223-265, Nueva York: Guilford Press.

American Psychiatric Association (1994). Manual diagnóstico y estadistico de los trastornos mentales: DSM-IV. Barcelona: Masson, 1995.

Baños, R., Belloch, A. y Perpiñá, C. (1990). Selfconsciousness scale: A study of Spanish housewives. Psychological Reports, 66, 771774.

Beck, A.T., Ward, C.M., Mendelson, M., Mock, J. y Erbaugh, J. (1961). An inventory for measuring depression. Archives of General Psychiatry, 4, 561-571.

Beck, A.T. (1976). Cognitive therapy and the emotional disorders. New York: Meridian.

Beck, A.T. (1987) Cognitive models of Depression. Journal of Cognitive Psychotherapy, 1, 5-37

Beck, A.T. (1991). Cognitive Therapy: A 30-year retrospective. American Psychologist, 46, 368375.

Beck, A.T. y Emery, G. (1985). Anxiety and phobias: A cognitive perpective. New York: Basic Books.

Beck, A.T., Rush, A.J., Shaw, B.F. y Emery, G. (1979). Cognitive Therapy of Depression. New York: Guilford Press (Trad. esp. en Bilbao: Desclée de Brower, 1983).
Belloch, A. (1990). Adaptación castellana del Inventario de pensamientos automáticos negativos (ATQ) de Hollon y Kendal. Edición experimental con autorización de los autores. Manuscrito no pulbicado.

Blaney, P.H. (1986). Affect and memory: A review. Psychological Bulletin, 99, 229-246.

Bower, G.H. (1981). Mood and memory. American Psychologist, 36, 129-148.

Bradley, B.P. y Mathews, A. (1983). Negative selfschemata in clinical depression. British Journal of Clinical Psychology, 22, 173-181.

Bradley, B.P. y Mathews, A (1988). Memory bias in recovered clinical depressives. Cognition and emotion, 2, 235-246

Brewin, C.R. (1988). Cognitive Foundations of Clinical Psychology. Hove: LEA.

Brewin, C.R., Smith, A.J., Power, M. y Furnham, A. (1992). State and trait differences in depressive self-perceptions. Behaviour Research Therapy, 30, 555-557.

Challis, B.H. y Krane, R.V. (1988). Mood induction and the priming of semantic memory in a lexical decision task: Asymmetric effects of elation and depression. Bulletin of the Psychonomic Society, 26, 309-312.

Clark, D.M.,Teasdale, J.D., Broadbent, D.E. y Martin, M. (1983). Effects of mood on lexical decisions. Bulletin of the Psychonomic Society, 21, 175-178.

Conde, V., Esteban, T. y Useros, E. (1976). Revisión crítica de la adaptación castellana del cuestionario de Beck. Revista de Psicología General y Aplicada, 31, 369-497.

Craik, F.I.M. y Lockhart, R.S. (1972). Levels of processing: A framework for memory research. Journal of Verbal Learning and Verbal Behaviour, 11, 671-684.

Craik, F.I.M. y Tulving, E. (1975). Depth of processing and retention of words in episodic memory. Journal of Experimental Psychology: General, 104, 268-294.

Davis, H. (1979a). Self-reference and the enconding of personal information in depression. Cognitive Therapy and Research, 3, 98-110.

Davis, H. (1979b). The self-schema and subjective organization of personal information in depression. Cognitive Therapy and Research, 3, 415-425.

Davis, H y Unruh, W.R. (1981). The development of the self-schema in adult depression. Journal os Abnormal Psychology, 90, 125-133.

Derry, P.A. y Kuiper, N.A. (1981). Schematic processing and self-reference in clinical depression. Journal of Abnormal Psychology, 90, 286-297. 
Ellis, H. C. y Ashbrook, P. W. (1988). Resource allocation model of the effects of depressed mood states on memory. En K. Fiedler and J. Forgas (Eds.), Affect, cognition and social behavior (pp.25-43). Gottingen: Hogrefe.

Ellis, H.C., Thomas, R.L. y Rodriguez, I.A. (1984). Emotional mood states and memories: Elaborative encoding, semantic processing, and cognitive effort. Journal of Experimental Psychology: Learning, Memory, and Cognition, 10, 470-482.

Ferguson, T.J., Rule, G.R.y Carlson, D. (1983). Memory for personally relevant information. Journal of Personality and Social Psychology, 44 251-261.

Foster, K.I. (1978). accesing the mental lexicon. En E. Walker (Ed.), Explorations in the Biology of Language. Montgomery: Bradford Books.

Gotlib, I. H. McLachlan, A.L. y Katz, A.N. (1988). Biases in visual attention in depressed and nondepressed individuals. Cognition and Emotiosn, 2, 185-200.

Greenberg, M.S. y Alloy, L.B. (1989). Depression versus anxiety: Processing of self-and otherreferent information. Cognition and Emotion, 3, 207-223

Hill, A.B. y Dutton, F. (1989). Depression and selective attention to self-esteem threatening words. Personality and Individual Differences, 10, 915-917.

Hollon, S.D. y Kendall, P.C. (1980). Cognitive selfstatements in depression: Developement of an automatic thoughts questionaire. Cognitive Therapy and Research, 4, 383-395.

Ingram, R.E., Kendall, P.C., Smith, T.W., Donnell, C. y Ronan, K. (1987). Cognitive specificity in emotional distress. Journal of Personality and Social Psychology, 53, 734-742.

Keenan, J.M. y Baillet, S.D. (1980). Memory for personally and socially significant events. En R.S. Nickerson (Ed.), Attention and performance, Vol. 8 (pp. 651-669). Hillsdale, NJ: LEA

Klieger, D.M. y Cordner, M.D. (1990). The Stroop task as measure of construct accessibility in depression. Personality and Individual Differences, 11, 19-27.

Kuiper, N.A. y MacDonald, M.R. (1982). Self and other perceptios in mild depressives. Social Cognition, 1, 223-239.

Kuiper, N.A. y MacDonald, M.R. (1983). Schematic processing in depression: The self-consensus bias. Cognitive Therapy and Research, 7, 469484.

Kuiper, N.A. y Rogers, T.B. (1979). Encoding of personal information: Self-other differences. Journal of Personality and Social Psychology, 37, 499-514.
Kuiper, N.A., Derry, P.A. y MacDonald, M.R. (1982). Self-reference and person perception in depression: A social cognition perspective. En G. Weary y H.L. Mirels (Eds.), Integration of Clinical and Social Psychology ((pp. 79-103). New York: Oxford University Press.

Kuiper, N.A., MacDonald, M.R. y Derry, P.A. (1983). Parameters of a depressive self-schema. En J. Suls y A.G. Greenwald, A.G. (Eds.), Psychological perspectives on the self (Vol. 2). Hillsdale, N.J.: Erlbaum.

Kuiper, N.A., Olinger, L.J., MacDonald, M.R. y Shaw, B.F. (1985). Self-schema processing of depressed and nondepressed content: The effects of vulnerability to depression. Social Cognition, 3, 77-93.

Kuiper, N.A., Olinger, L.J (1986). Dysfunctional attitudes and a self-worth contingency model of depression. En P.C. Kendall (Ed.) Advances in cognitive Behavioral Research and Therapy, vol.5, págs. 115-142. Nueva York: Academic Press.

Lewinshon, P.M., Mischel, W., Chaplin, W. y Barton, R. (1980). Social competence and depression: The role of illusory self-perceptions. Journal of Abnormal Psychology, 89, 203-212.

MacDonald, M.R. y Kuiper, N.A. (1985). Efficiency and automataicity of self-schema processing in clinical depresives. Motivation and Emotion, 9, 171-184.

MacDonald, M.R. y Kuiper, N.A. (1984). Selfschema decision consistency in clinical depressives. Journal of Social and Clinical Psychology, 2, 264-272.

MacLeod, C., Tata, P. y Mathews, A. (1987). Perception of emotionally valenced information in depression. British Journal of Clinical Psychology, 26, 67-68.

Markus, H. (1977). Self-schemata and processing information about the self. Journal of Personality and Social Psychology, 35, 63-78.

Markus, $H$. y Nurius, $P$ (1987). Possible selves: The interface between motivation and the self concept. En K. Yardley y T. Honess (Eds.), Self and Identity (pp. 157-172) New York: Wiley.

Mathews, A. y MacLeod, C. (1994). Cognitive approaches to emotion. Annual Review of Psyhology, 45, 25-50.

Matt, G., Vázquez, C. y Campbell, K.W. (1992). Mood-congruent recall of affectively toned stimuli: A meta-analytic review. Clinical Psychology Review, 12, 227-255.

McCaul, K.D. y Maki, R.H. (1984). Self-reference versus desirability ratings and memory for traits. Journal of Personality and Social Psychology, 47, 953-955. 
Mueller, J-H. (1982). Self-awareness and access to material rated as self-descriptive or nondescriptive. Bulletin of the Psychonomic Society, 19, 323-326.

Myers, J.F. (1984). Schematic processing and selfreference during contiguous periods of clinical depression and symptomatic remission. Tesis Doctoral. Departamento de Psiología. Universidad de Calgary

Riskind, J.H. (1989). The mediating mechanisms in mood and memory: A cognitive-priming formulation. Journal os Sical Behavior and Personality, 4, 173.184.

Roth, D. y Rhem, L.P. (1980). Relationships among self-monitoring processes, memory, and depression. Cognitive therapy and Research, 4 149-157.

Ruiz-Caballero, J. A. y Bermúdez, J. (1993). Estado de ánimo depresivo, decisión léxica y percepción de información emocional. Boletin de Psicología, 39, 67-76.

Sanz, J. (1992). Procesos cognitivos en la ansiedad y en la depresión. Tesis doctoral. Universidad Complutense de Madrid.

Sanz, J. (1994). Autoesquemas depresivos y ansiosos: se busca una estructura. Boletín de Psicología, $n^{\circ}$ 42: 75-107.
Segal Z.V. y Swallow, S.R. (1994). Cognitive assessment of unipolar depression: measuring products, processes and structures. Behavior Research and Therapy, 32, 147-158.

Scheier, M.F. y Carver, C.S. (1985). The selfconsciousness scale: a revised version for use with general populations. Journal of Applied Ssocial Psychology, 15, 687-699.

Seisdedos, N. (1988). Cuestionario de Ansiedad Estado-Rasgo. Adaptación española. Madrid, TEA ediciones.

Singer, J. L. y Salovey, P (1988). Mood and memory: evaluating the network theory of affect. Clinical Psychology Reviw, 8, 211-251.

Spielberger, C.D., Gorsuch, R.L. y Lushene, R.E. (1970) Manual for the State-Trait Anxiety Inventory.Palo Alto, CA: Consulting Psychologist Press.

Vázquez, C y Alloy, L.B. (1987). Schematic memory processes for self- and other-referent information in depression versus anxiety: A signal detection analysis. Northwestern University, Evanston.

Williams, J.M.G., Watts, F.N., MacLeod, C. y Mathews, A. (1988). Cognitive psychology and emotional disorders. New York: Wiley.

Wine, J.D. (1971). Test anxiety and directions of attention. Psycological Bulletin, 76, 92-104. 


\section{APÉNDICE}

Adjetivos descriptivos utilizados como estímulos en la tarea de procesamiento semántico autorreferente

\begin{tabular}{|c|c|c|}
\hline Positivos & Negativos & Neutros. \\
\hline Activo/a & Abatido/a & Ambicioso/a \\
\hline Afortunado/a & Aburrido/a & Artista \\
\hline Agradable & Acabado/a & Astuto/a \\
\hline Alegre & Agobiado/a & Atento/a \\
\hline Animado/a & Amargado/a & Celoso/a \\
\hline Atrevido/a & Apagado/a & Conservador/a \\
\hline Bueno/a & Apenado/a & Creativo/a \\
\hline Confiado/a & Cobarde & Educado/a \\
\hline Contento/a & Desdichado/a & Envidioso/a \\
\hline Decidido/a & Desesperado/a & Grosero/a \\
\hline Divertido/a & Desgraciado/a & Honesto/a \\
\hline Eficaz & Enfermizo/a & Informal \\
\hline Encantador/a & Fracasado/a & Lento/a \\
\hline Enérgico/a & Indeciso/a & Listo/a \\
\hline Feliz & Indefenso/a & Modesto/a \\
\hline Fuerte & Infeliz & Natural \\
\hline Gracioso/a & Lloroso/a & Normal \\
\hline Interesante & Miserable & Ocupado/a \\
\hline Optimista & Nervioso/a & Presumido/a \\
\hline Sano/a & Pesaroso/a & Racional \\
\hline Satisfecho/a & Retraido/a & Simple \\
\hline Simpático/a & Sensible & Tolerante \\
\hline Sociable & Solitario/a & Tonto/a \\
\hline Tranquilo/a & Sombrio/a & Trabajador/a \\
\hline Valiente & Triste & Vago/a \\
\hline
\end{tabular}

